\title{
CUTANEOUS LEIOMYOSARCOMA: A CLINICAL, DERMOSCOPIC, PATHOLOGIC CASE STUDY
}

\author{
V. De Giorgi ${ }^{1,}$ *, F. Scarfi ${ }^{1}$, F. Silvestri ${ }^{1}$, P. Maida ${ }^{1}$, A. Gori ${ }^{2}$, L. Trane ${ }^{1}$, D. Massi ${ }^{3}$ \\ ${ }^{1}$ Department of Dermatology, University of Florence, Florence 50100, Italy \\ ${ }^{2}$ Cancer Research "Attilia Pofferi” Foundation, Pistoia 51100, Italy \\ ${ }^{3}$ Department of Human Pathology and Oncology, University of Florence, Florence 50100, Italy
}

Cutaneous leiomyosarcoma is a rare malignant mesenchymal tumor of smooth muscle origin of unknown etiology. Leiomyosarcomas located on the skin include superficial leiomyosarcomas and metastatic leiomyosarcomas. Superficial leiomyosarcomas represent 7-10\% of all cases of leiomyosarcoma and are subdivided into cutaneous and subcutaneous lesions based on skin location. We report a case of an 81-year-old woman who presented a slow growing tender single nodule on the left thigh and describe its clinical and dermoscopic features.

Key Words: cutaneous leiomyosarcoma, pathology, immunohistochemistry, dermoscopic study.

An 81-year-old woman was referred to our clinic for the presence of a slow growing tender single nodule on the left thigh. The patient reported that the tumor had started to grow slowly one year ago with no symptoms. The patient was in apparently good health condition in consideration of her age.

On physical examination the lesion appeared as a firm, skin-colored, sessile nodule on the left thigh (Fig. 1). There were no palpable lymph nodes on the left groin. Dermoscopic examination revealed an aspecific non-pigmented pattern with the presence of arborizing vessels and spread telangiectasias on a background of whitish area that resembled the dermoscopic pattern of non-pigmented basal cell carcinoma. In addition, there was a thicker vessel on the peripheral area of the lesion. The vessel partially surrounded the lesion (Fig. 1). On the basis of this raveled the diagnosis of cutaneous leiomyosarcoma (Fig. 2). Morphological diagnosis was supported by immunohistochemical positivity of tumor cells with smooth muscle markers (smooth muscle actin $1 \mathrm{~A} 4$, common muscle actin HHF35 and desmin). The patient was surgically retreated with a wide local excision, with 2-cm lateral margins. After 24 months of follow up she resulted negative for the presence of local recurrence and with no evidence of disease recurrence or metastasis.

\section{DISCUSSION}

Cutaneous leiomyosarcoma is a rare malignant mesenchymal tumor of smooth muscle origin of unknown etiology [1]. Leiomyosarcomas located on the skin include superficial leiomyosarcomas and metastatic leiomyosarcomas. Superficial leiomyosarcomas
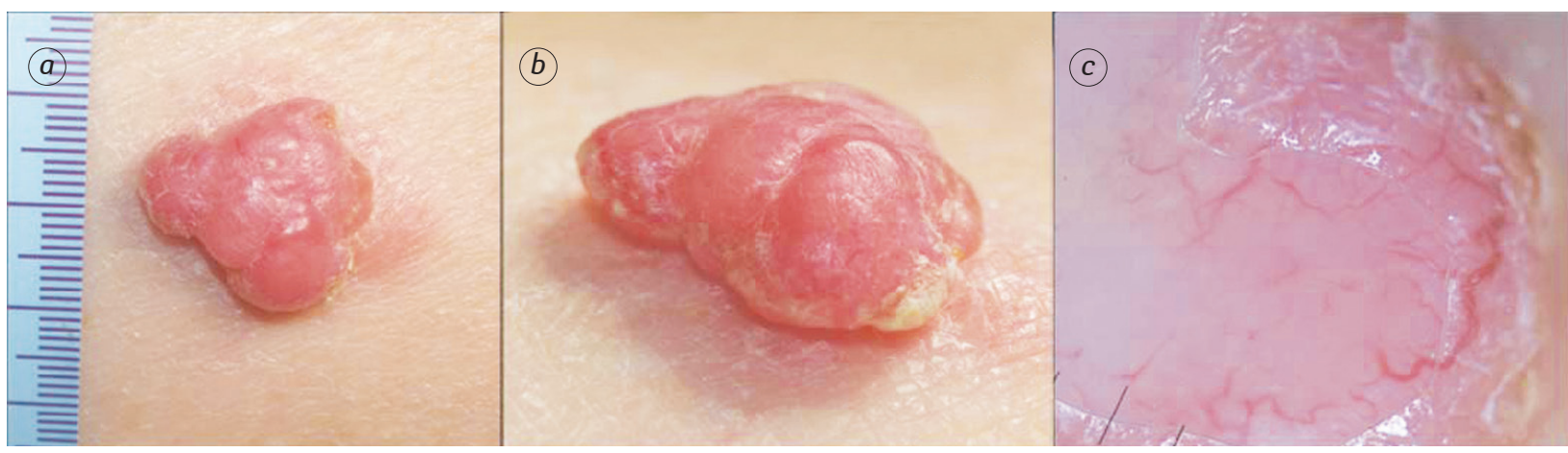

Fig. 1. The nodule of approximately $2 \mathrm{~cm}$ in diameter appears firm, skin-colored and sessile $(a, b)$. Dermoscopic examination revealed an aspecific non pigmented pattern with the presence of arborizing vessels and spread telangiectasias on a background of whitish area. A thicker vessel that partially surrounded the lesion is present on the peripheral area of the lesion $(c)$

undefined clinical and dermoscopic aspect of the lesion we decided to perform an excisional biopsy with histological examination.

The histological examination showed a dermally based proliferation of spindle cells with blunt-ended, "cigar-shaped" nuclei and multiple mitotic figures that

Submitted: September 30, 2018.

Correspondence: $\quad$ E-mail: vincenzo.degiorgi@unifi.it represent $7-10 \%$ of all cases of leiomyosarcoma and are subdivided into cutaneous and subcutaneous lesions based on skin location [2, 3]. The extremities are the most common body locations, particularly the face and neck area and the legs [4]. Histologically, cutaneous leiomyosarcomas are situated primarily in the dermis and extend occasionally into the subcutaneous fat tissue. These tumors are thought to arise from the arrector pili muscle of the hair follicle [1]. The develop- 


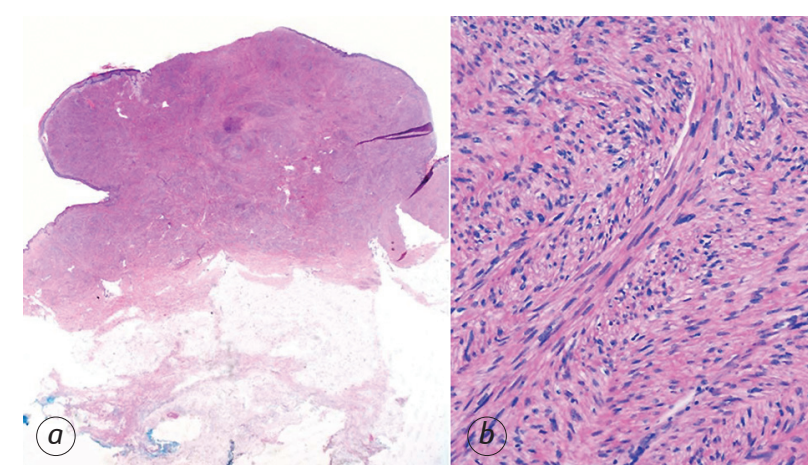

Fig. 2. Cutaneous leiomyosarcoma: a - purely dermal cutaneous leiomyosarcoma showing a polypoid architecture (original magnification $\times 5$ ); $b$ - the tumor is composed of long intersecting, occasionally perpendicular, fascicles of smooth muscle cells having an elongated shape, blunt-ended nuclei, and eosinophilic cytoplasm (original magnification $\times 20$ )

ment of metastases from cutaneous leiomyosarcoma is exceptional but these tumors may show aggressive biologic potential that can determinate a significant local destruction.

Clinically, a cutaneous leiomyosarcoma usually appears as a skin colored soft node with no specific features, therefore the potential differential diagnosis is with a number of lesions including common benign lesions such as cysts, lipomas, fibromas, persistent insect bites, pyogenic granuloma, neurofibromas, dermatofibromas, and intradermal nevi. Moreover, a cutaneous leiomyosarcoma can be misdiagnosed also with malignant cutaneous lesions such as basal cell carcinoma, squamous cell carcinoma, amelanotic melanoma and cutaneous metastasis of an internal malignancy. In our case, even if the clinical appearance of the lesion did not allow for the diagnosis, the dermoscopic pattern brought us to suspect a malign lesion. The dermoscopic pattern of our lesion could be matched with the diagnosis of either basal cell carcinoma or amelanotic melanoma. Therefore the undefined pattern should suggest a diagnosis of a malignant lesion that requires a prompt surgical treatment.

\section{REFERENCE}

1. Massi D, Franchi A, Alos L, et. al. Primary cutaneous leiomyosarcoma: clinicopathological analysis of 36 cases. Histopathology 2010; 56: 251-62.

2. Starling J 3 ${ }^{\text {rd }}$, Coldiron BM. Mohs micrographic surgery for the treatment of cutaneous leiomyosarcoma. J Am Acad Dermatol 2011; 64: 1119-22.

3. De Giorgi V, Sestini S, Massi D, et al. Superficial cutaneous leiomyosarcoma: a rare, misleading tumor. Am J Clin Dermatol 2008; 9: 185-7.

4. Torres T, Oliveira A, Sanches M, Selores M. Superficial cutaneous leiomyosarcoma of the face: report of three cases. J Dermatol 2011; 38: 373-6. 